\title{
Prix Jean Perrin 2012 (popularisation de la science) : Denis Savoie
}

Denis Savoie dirige le planétarium et le département astronomie du Palais de la découverte. Il est aussi chercheur associé au département Systèmes de référence temps espace (SYRTE) de l'Observatoire de Paris et membre correspondant de I'Académie Internationale d'Histoire des Sciences.

Il fut président de la Commission des cadrans solaires de la Société astronomique de France durant 20 ans. Flammarion moderne, il a consacré sa carrière à la diffusion des connaissances astronomiques. Spécialiste de la théorie et de I'histoire des cadrans solaires, Denis Savoie a publié deux ouvrages qui sont considérés comme des références en la matière : La gnomonique et Les cadrans solaires. À ceux-là, on peut ajouter sa Cosmographie (Belin, Pour la Science).

En matière de cadrans solaires, son œuvre témoigne de sa renommée internationale : il a conçu en particulier la nef solaire, cadran solaire monumental installé sur l'aire de Tavel de l'autoroute A9 (1993) (fig. 1), le cadran solaire de la Bibliothèque d'Alexandrie (2004) et le gigantesque cadran tracé sur le barrage de Castillon (2009).
Denis Savoie est aussi impliqué dans la défense de la science, au sens noble du terme. Son inlassable action auprès des jeunes, des étudiants, des enseignants et du grand public permet de rendre accessible au plus grand nombre et de façon rigoureuse une astronomie bien mal traitée dans l'enseignement, et dont la pollution lumineuse de nos grandes villes nous éloigne chaque jour un peu plus.

En récompensant Denis Savoie, le jury du prix Perrin a voulu, à travers lui, mettre l'accent sur l'importance du travail des médiateurs scientifiques tels qu'ils ont été mis en place au Palais de la découverte dès sa création, et qui devraient avoir une place dans toutes les structures de diffusion de la culture scientifique.

\section{Discours de Denis Savoie, lors de sa remise de prix au Palais de la découverte, le 16 mai 2013}

Je suis très honoré ce soir de recevoir le prix Jean Perrin que me décerne la Société Française de Physique et ce, d'autant plus que ce prix porte le nom du fondateur de l'établissement dans lequel j'ai effectué l'essentiel de ma carrière, le Palais de la découverte. Pour les astronomes, Jean Perrin est un savant qui a marqué la discipline puisque, en plus du Palais, il a fondé l'Institut d'astrophysique de Paris et l'Observatoire de Haute-Provence !

Je me suis souvent demandé ce que penserait Jean Perrin s'il revenait aujourd'hui au Palais. Je suppose qu'il serait d'abord surpris, voire très surpris ! Surpris d'abord que ce Palais des sciences existe toujours 76 ans après sa fondation ; surpris encore plus en le visitant, car certaines salles lui sembleraient quasiment dans le même état qu'en 1937, ce qui pour certains est très inquiétant. En ce qui concerne le planétarium, je ne crois pas qu'il serait complètement dépaysé ; concrètement, il est vraisemblable que le discours que l'on y tient aujourd'hui est peu différent de l'époque où le planétarium se trouvait sur les bords de Seine. Certains, bien sûr, s'en offusqueront aussi : combien de fois m'a-t-on reproché de ne pas montrer au public enfin de l'astronomie moderne, c'est-à-dire montrer le ciel en rayons X, en rayons gamma, plutôt que de s'accrocher à de vieilles antiennes... Et, en fait, ce reproche pourrait être fait à bien d'autres disciplines du Palais ; ce serait oublier la mission de cet établissement depuis sa fondation : vulgariser la science fondamentale. Comment comprendre et mesurer l'importance des dernières découvertes en astronomie aujourd'hui, si on ne connaît pas les bases ? Ces bases en astronomie, cette astronomie qui, pour paraphraser Kepler, " est fille du temps ", sont en dehors du temps justement : comme les lois de l'électrostatique en physique sont immuables, les lois qui régissent les mouvements des astres le sont aussi à l'échelle humaine. Ce sont ces bases que chaque jour nous transmettons au public. Et c'est peut-être parce que le public qui fréquente le Palais reçoit ce discours, je devrais dire l'outil indispensable à la compréhension du monde, qu'il se dit parfois " ah d'accord, j'ai compris pourquoi ».

Mais l'astronomie au Palais ne se résume pas au planétarium; la salle des planètes, le balcon de cosmologie, qui eux présentent l'état le plus actuel de l'astronomie et de l'astrophysique, participent activement à faire découvrir au public cette science qu'on dit la plus ancienne. Heureusement aujourd'hui, la médiation au Palais de la découverte ne se résume pas à un discours bien rôdé que l'on déverserait à longueur de journée ; la conception des salles, leur contenu scientifique, la façon d'interagir, tout comme les expositions temporaires, tout ceci fait et doit faire partie du métier de médiateur.

Et il faut en convenir, je pense que le Palais de ce début du $\mathrm{XXI}^{\mathrm{e}}$ siècle est bien mieux que celui des années 80, époque où je suis arrivé. Cela tient essentiellement à la médiation scientifique, qui a beaucoup évolué et qui s'est considérablement améliorée : elle est désormais délivrée par des personnes au coefficient de vétusté beaucoup moins élevé qu'à une époque où des chargés d'exposé en blouse blanche hantaient les salles d'expériences... Le Palais s'est rajeuni, les médiateurs ont une formation scientifique bien plus sérieuse qu'autrefois; ils ne sont plus cantonnés à une oralité répétitive. Jean Perrin pourrait être fier que l'idée qu'il a eue à l'époque du Front Populaire, cette idée de partager et de faire découvrir la science aux citoyens grâce à des expériences et 


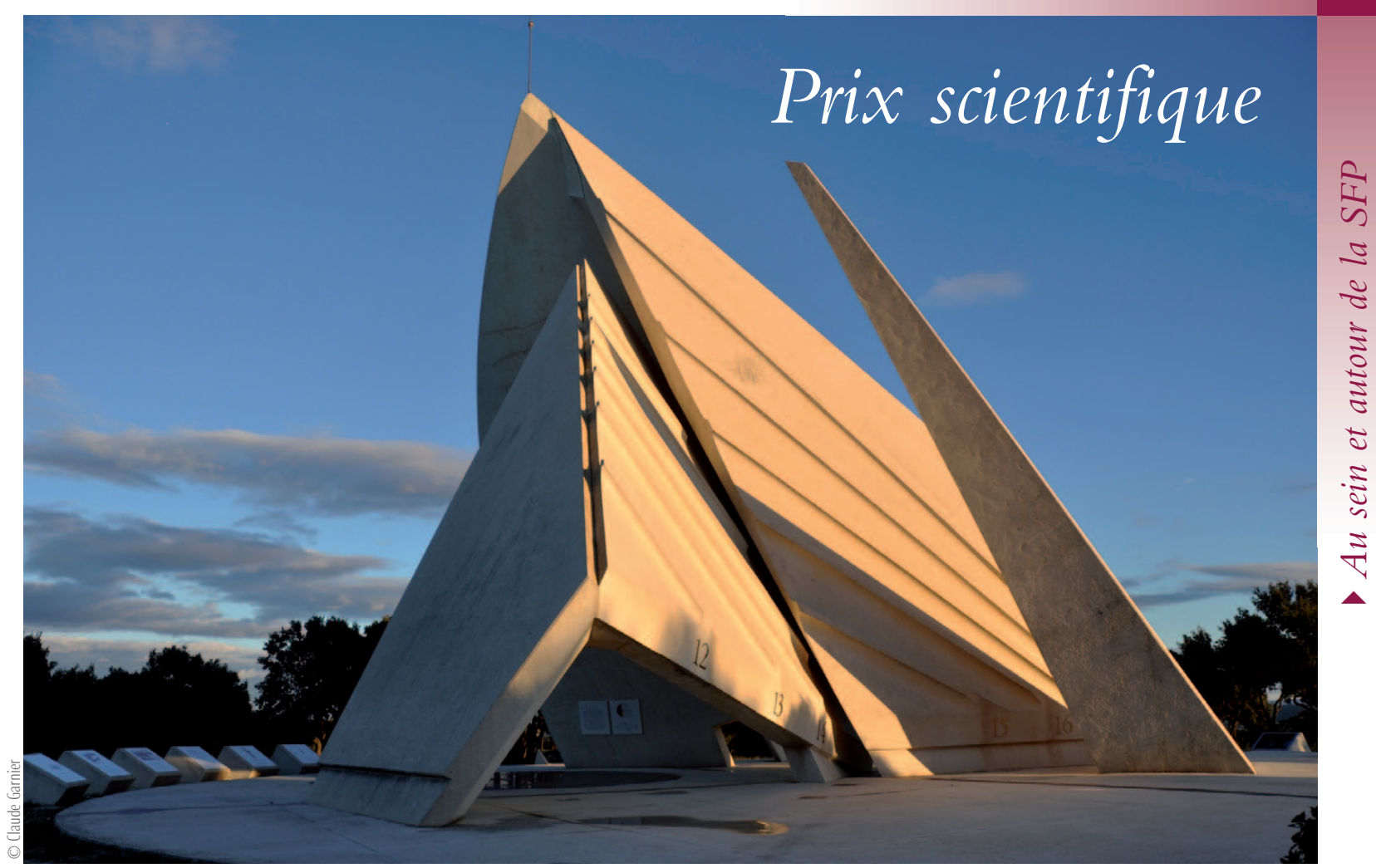

1. Nef solaire de Tavel, imaginée par la sculpteur Odile Mir. Ce cadran solaire monumental a été calculé par Denis Savoie en 1993 ; il se trouve sur l'aire nord de Tavel sur l'autoroute A9, près d'Avignon. De nombreuses explications astronomiques et historiques complètent cette remarquable réalisation.

des médiateurs, n'a pas pris une ride. Elle enthousiasme encore un public jeune et moins jeune qui fréquente assidûment, années après années, depuis donc plus de 76 ans, ce Palais qui pourtant a connu et connait encore des vicissitudes, à un point tel qu'on pourrait graver sur son fronton la devise Fluctuat nec mergitur, il ballote mais ne sombre pas!

Je ne pourrai pas dire combien j'ai fait de séances de planétarium et d'exposés d'astronomie en un quart de siècle : des milliers, sans doute. Jamais je n'aurais pu mener à bien cette médiation, organiser le département d'astronomie, publier des ouvrages et poursuivre parallèlement depuis dix-sept ans mes recherches à l'Observatoire de Paris, au sein de l'équipe Histoire du département Temps-Espace, le Syrte, sans mes chers collègues que je voudrais associer ce soir à ce prix Jean Perrin. Je tiens donc à remercier ici Marc Goutaudier, Rémi Tropé, Sébastien Fontaine, Johan Kieken, Stéphane Fay, Alain Redding et Philippe Thebault. Sans eux, rien de tout cela n'aurait été possible. Et, vous l'avez compris, si le Palais de la découverte permet tant d'activités dans la médiation, c'est qu'on y jouit d'une grande liberté et d'une grande confiance : ce sont des atouts qu'il faut préserver, car ils sont précieux.

Le prix Jean Perrin récompense la popularisation de la science ; cette popularisation n'a pas eu dans mon cas comme seul vecteur le Palais de la découverte. Très tôt j'ai eu la chance de rencontrer un maitre qui m'a enseigné la gnomonique, une sorte de "microniche " dans le champ de l'astronomie. Et celle-ci est devenue pour moi un axe majeur pour faire découvrir une astronomie peu connue, celle de la mesure du temps, qui regroupe de la technique et de l'histoire, voire de l'art, mais je préfere le mot esthétisme. Et j'avoue que j'ai pris un plaisir immense à réaliser des cadrans solaires, grands ou petits, en France et à l'étranger. À chaque fois, ces cadrans qui paraissent assez simples ont été l'occasion d'expliquer au public que derrière eux se cachent des mathématiques, de l'astronomie, de l'histoire des sciences, voire de la philosophie lorsque l'on s'attache aux devises souvent latines qui évoquent la fuite du temps ou la brièveté de la vie. J'ai toujours été attentif à une médiation pragmatique, pour ne pas dire concrète, de l'astronomie et de l'histoire de l'astronomie ; et les cadrans solaires constituent un vecteur, un moyen d'intéresser le public, alors que l'astronomie est une science assez impalpable, où faire des expériences comme en chimie ou en physique est très difficile.

À une époque pas si éloignée, on aurait pu me demander de dresser l'horoscope du Palais de la découverte. Quoi de plus facile avec le planétarium en plus... Que va devenir le Palais ? Va t-il "surfusioner " avec le Grand Palais après avoir fusionné avec la Cité des Sciences, et se trouver alors dans un état métastable ? À cela je répondrai, comme le ferait un astrologue, toujours habile, que les astres inclinent mais ne déterminent pas... Il est cependant facile d'entrevoir que dans une France où la désaffection des jeunes pour les carrières scientifiques devient un réel sujet de préoccupation pour les politiques, le Palais de la découverte (et plus généralement Universcience) a plus que jamais une légitimité forte dans la diffusion des savoirs. Pour conclure, je ferai mienne cette prédiction d'un grand astrophysicien français, Jean-Claude Pecker : «La culture scientifique est partie intégrante de toute culture. Sans sa composante scientifique, toute culture est vouée à l'échec. "

Denis Savoie 